\title{
CONTROLADOR EM MODO DUAL ADAPTATIVO ROBUSTO PARA PLANTAS COM GRAU RELATIVO UNITÁRIO: PROVA DE ESTABILIDADE
}

\author{
Caio D. Cunha* \\ dorneles@ufrnet.br
}

\author{
Aldayr D. Araújo* \\ aldayredca.ufrn.br
}

\author{
Francisco C. Mota ${ }^{\dagger}$ \\ motaedca.ufrn.br \\ * Departamento de Engenharia Elétrica - UFRN \\ 59.072-970 Natal-RN, fone 843215 3732-fax 8432153731 \\ ${ }^{\dagger}$ Departamento de Engenharia de Computação e Automação - UFRN \\ 59.072-970 Natal-RN, fone 843215 3771-fax 8432153738
}

\section{RESUMO}

Neste artigo é apresentada uma prova de estabilidade para o controlador em Modo Dual Adaptativo Robusto (DMARC) para plantas com grau relativo unitário. O DMARC é um controlador que interpola as estratégias de Controle Adaptativo por Modelo de Referência (MRAC) e o Controle Adaptativo por Modelo de Referência e Estrutura Variável (VSMRAC) com o intuito de incorporar as vantagens de desempenho transitório de um VS-MRAC, com as propriedades de regime permanente de um MRAC convencional. Desta forma, deseja-se obter um sistema de controle robusto a incertezas paramétricas e perturbações externas, com desempenho rápido e pouco oscilatório durante o transitório e um sinal de controle suave em regime permanente.

PALAVRAS-CHAVE: Controle em Modo Dual, Controle Adaptativo, Sistemas com Estrutura Variável.

ARTIGO CONVIDADO:

Versão completa e revisada de artigo apresentado no SBAI-2005

Artigo submetido em 21/06/2006

1a. Revisão em 14/08/2006

2a. Revisão em 13/12/2006

3a. Revisão em 23/02/2007

Aceito sob recomendação do Editor Convidado

Prof. Osvaldo Ronald Saavedra Mendez

\section{ABSTRACT}

In this paper the stability proof to the Dual Mode Adaptive Robust Control (DMARC) applied to plants with relative degree one is presented. The DMARC is a control strategy which interpolates the Model Reference Adaptive Control (MRAC) and the Variable Structure Model Reference Adaptive Control ( VS-MRAC). The main idea is to develop a robust controller to parametric uncertainties and external disturbances with good transient characteristics (fast response and small oscillations) as in a VS-MRAC and good steadystate characteristic (smooth control signal) as in a conventional MRAC.

KEYWORDS: Dual Mode Control, Adaptive Control, Variable Structure Systems.

\section{INTRODUÇÃO}

O Controlador DMARC teve seu início a partir da idéia proposta por Hsu e Costa (1989). Os autores utilizaram uma expressão que, dependendo da escolha de um parâmetro, podia se comportar como o Controlador MRAC ou como o Controlador VS-MRAC. Essa expressão, para a lei de adaptação dos parâmetros do controlador, foi utilizada para justificar que o VS-MRAC pode ser visto como um controlador adaptativo sem memória e com aprendizado instantâneo (ver Hsu 
e Costa (1989) e (1990)).

A partir da teoria de controle binário desenvolvida por Emelyanov (1987), Hsu e Costa (1990) propuseram um Controlador Binário Adaptativo por Modelo de Referência (BMRAC) para plantas com grau relativo $\left(n^{*}\right)$ igual a 1 e depois generalizado em Hsu e Costa (1994), para plantas com grau relativo $\left(n^{*}\right)$ arbitrário. No B-MRAC é utilizada uma lei gradiente de adaptação de alto ganho com projeção, a qual com um parâmetro fixo suficientemente alto tende ao VS-MRAC.

No controlador MRAC convencional, que usa leis integrais de adaptação, a saída da planta segue um modelo de referência especificado (Ioannou e Sun (1996), Åström e Wittenmark (1995), Sastry e Bodson (1989) e Narendra e Annaswamy (1988)). Mesmo com as modificações para aumentar a robustez do algoritmo convencional (fator $\sigma$, normalização, etc.) (Ioannou e Sun, 1996) em geral o transitório é lento e oscilatório.

No VS-MRAC, utiliza-se a estrutura de controle por modelo de referência do MRAC e leis chaveadas, como nos sistemas com estrutura variável, para o sinal de controle (Hsu e Costa (1989), Hsu, Araújo e Costa (1994), Hsu, Lizarralde e Araújo (1997), Cunha e outros (2003)). Apesar do bom desempenho transitório, em geral temos a presença do fenômeno de "chattering".

O Controlador em Modo Dual Adaptativo Robusto propõe uma ligação entre o VS-MRAC e o MRAC convencional. O objetivo é conseguir um sistema robusto, com desempenho rápido e pouco oscilatório (características do VS-MRAC), e sinal de controle suave em regime permanente (característica do MRAC).

Uma versão inicial do controlador DMARC, utilizando a lei de adaptação proposta em Hsu e Costa (1989), foi apresentada nos artigos Cunha e outros (2005) e Mota e Araújo (2002). Nesses trabalhos, o parâmetro que dita a transição entre as duas estratégias de controle (MRAC e VS-MRAC) é ajustado em tempo real, utilizando a lógica fuzzy.

Neste trabalho, é proposta uma modificação da lei de adaptação inicial do DMARC utilizada nos trabalhos anteriores (Cunha e Araújo(2004) e Cunha e outros (2005)). Foi acrescentado um termo na lei de adaptação para eliminar os termos de sinais indefinidos que aparecem na derivada da função de Lyapunov.

Esse artigo é organizado como segue. Na seção 2 é dada a formulação do sistema a ser controlado. Os controladores MRAC convencional, VS-MRAC, B-MRAC e DMARC são apresentados nas seções 3, 4, 5 e 6 respectivamente. A análise de estabilidade é feita na seção 7 . Na seção 8 é mostrada uma das possíveis formas de ajuste do DMARC, a qual é uti- lizada nas simulações da seção 9.

\section{FORMULAÇÃO DO PROBLEMA}

Considere uma planta linear com parâmetros incertos monovariável e invariante no tempo com função de transferência

$$
W(s)=k_{p} \frac{N_{p}(s)}{D_{p}(s)}
$$

com entrada $u$ e saída $y$ e o modelo de referência tendo a entrada $r$ e a saída $y_{m}$ caracterizado pela função de transferência

$$
M(s)=k_{m} \frac{N_{m}(s)}{D_{m}(s)}
$$

Tem-se como objetivo determinar $u$ tal que o erro de saída

$$
e_{0}=y-y_{m}
$$

tenda a zero assintoticamente para condições iniciais arbitrárias e sinais de referência $r(t)$ uniformemente limitados e contínuos por partes.

As seguintes hipóteses convencionais são feitas (Sastry e Bodson (1989), pag. 103-104):

a. A planta é controlável e observável com $\operatorname{grau}\left[D_{p}(s)\right]=$ $n$ e $\operatorname{grau}\left[N_{p}(s)\right]=m, n$ e $m$ conhecidos;

b. $\operatorname{sgn}\left(k_{p}\right)=\operatorname{sgn}\left(k_{m}\right)$, positivos, sem perda de generalidade;

c. $N_{p}(s)$ é Hurwitz ( $W(s)$ é de fase mínima);

d. O modelo de referência é estritamente real positivo (ERP) e tem o mesmo grau relativo da planta $\left(n^{*}=n-m=\right.$ $1)$;

e. Considera-se apenas a realimentação de saída da planta.

São usados os seguintes filtros de entrada e saída da planta

$$
\left\{\begin{array}{l}
\dot{v}_{1}=\Lambda v_{1}+g u \\
\dot{v}_{2}=\Lambda v_{2}+g y
\end{array}\right.
$$

sendo $v_{1}$ e $v_{2}$ pertencentes ao $\Re^{n-1}, g$ pertence ao $\Re^{n-1}$ e $\Lambda$ uma matriz estável pertencente ao $\Re^{(n-1) x(n-1)}$, escolhidos 
tal que o par $(\Lambda, g)$ é controlável e $N_{m}(s)=\operatorname{det}(s I-\Lambda)$. Define-se o vetor regressor como $\omega^{T}=\left[\begin{array}{llll}v_{1}^{T} & y & v_{2}^{T} & r\end{array}\right]$. O controle é, então, definido como

$$
u=\theta(t)^{T} \omega(t)
$$

onde $\theta(t)^{T}=\left[\theta_{v 1}^{T}(t) \theta_{n}(t) \theta_{v 2}^{T}(t) \theta_{2 n}(t)\right]$ é o vetor de parâmetros adaptativos (Ioannou e Sun (1996), pag. 384).

Sabe-se que, sob as hipóteses acima, existe um único vetor constante $\theta^{*}$ tal que a função de transferência da planta em malha fechada (com $\left.u=\theta^{*^{T}} \omega\right)$, de $r$ para $y$, é $M(s)$ (condição de "Matching"). Obviamente $\theta^{*}$ somente pode ser conhecido se a planta for conhecida .Quando isto não é o caso, $\theta(t)$ é adaptado até que $e_{0}(t) \rightarrow 0$ quando $t \rightarrow \infty$ e, sob alguma condição de riqueza de sinal, $\theta(t) \rightarrow \theta^{*}$ (Ioannou e Sun (1996), Pag. 177).

Seja $\left[A, b, h^{T}\right]$ uma realização mínima da planta e $\mathrm{x} \in$ $\Re^{n}$ o respectivo vetor de estado. Então, a planta com os filtros podem ser representados como

$$
\begin{gathered}
\dot{x}_{c}=A_{0} x_{c}+b_{0} u \\
y=h_{c}^{T} x_{c}
\end{gathered}
$$

onde $x_{c}^{T}=\left[x^{T}, v_{1}^{T}, v_{2}^{T}\right], x_{c} \in \Re^{3 n-2}$,

$$
\begin{gathered}
A_{0}=\left[\begin{array}{ccc}
A & 0 & 0 \\
0 & \Lambda & 0 \\
g h^{T} & 0 & \Lambda
\end{array}\right], \quad b_{0}=\left[\begin{array}{l}
b \\
g \\
0
\end{array}\right] \\
h_{c}^{T}=\left[\begin{array}{lll}
h^{T} & 0 & 0
\end{array}\right]
\end{gathered}
$$

Nota-se que $\omega=\Omega x_{c}+b_{r} r$ com

$$
\Omega=\left[\begin{array}{c}
\Omega_{c} \\
0
\end{array}\right], \quad \Omega_{c}=\left[\begin{array}{ccc}
0 & I & 0 \\
h^{T} & 0 & 0 \\
0 & 0 & I
\end{array}\right] \text { e } b_{r}=\left[\begin{array}{l}
0 \\
0 \\
0 \\
1
\end{array}\right]
$$

Tem-se, então,

$$
\begin{gathered}
\dot{x}_{c}=A_{c} x_{c}+\frac{b_{c}}{\theta_{2 n}^{*}}\left(u-\theta^{*^{T}} \omega\right)+b_{c} r \\
y=h_{c}^{T} x_{c}
\end{gathered}
$$

onde $\quad A_{c}=A_{0}+b_{0} \theta_{r}^{*^{T}} \Omega_{c}, \quad \theta_{r}^{*^{T}}=\left[\theta_{1}^{*} \ldots \theta_{2 n-1}^{*}\right]$, $b_{c}=\theta_{2 n}^{*} b_{0}, \theta_{2 n}^{*}=k_{m} / k_{p}>0$.
$\left[A_{c}, b_{c}, h_{c}^{T}\right]$ é uma realização não mínima e estável de M(s) (Sastry, (1984)), ou seja, o modelo de referência pode ser representado como

$$
\begin{gathered}
\dot{x}_{c m}=A_{c} x_{c m}+b_{c} r, \quad x_{c m} \in \Re^{3 n-2} \\
y_{m}=h_{c}^{T} x_{c m}
\end{gathered}
$$

Definindo o vetor de erro por $e=x_{c}-x_{c m}$ tem-se a seguinte equação de erro

$$
\begin{gathered}
\dot{e}=A_{c} e_{c}+\frac{b_{c}}{\theta_{2 n}^{*}}\left(u-\theta^{*^{T}} \omega\right) \\
e_{0}=h^{T} e
\end{gathered}
$$

e, na forma entrada saída,

$$
e_{0}=\frac{1}{\theta_{2 n}^{*}} M\left(u-\theta^{*^{T}} \omega\right)
$$

\section{MRAC CONVENCIONAL}

No MRAC o desempenho desejado para a planta é definido por um modelo de referência. A função de transferência para o modelo de referência deve ser definida com a mesma ordem do modelo nominal da planta. O objetivo do MRAC é que a planta siga o modelo (condição de “matching”).

Se os parâmetros da planta são conhecidos com exatidão, têm-se os valores dos parâmetros do controlador $\theta^{*}$ para os quais a saída da planta converge para a saída do modelo (Ioannou e Sun(1996), pag. 333). Porém, se esses parâmetros são desconhecidos ou conhecidos com incertezas, torna-se necessária uma adaptação para os parâmetros do controlador. No MRAC o sinal de controle é dado por

$$
u=\theta^{T} \omega
$$

e a lei original de adaptação por

$$
\dot{\theta}=-\gamma e_{0} \omega \quad, \quad \gamma>0
$$

No MRAC, o algoritmo é baseado na estimação dos parâmetros e contém leis de adaptação integrais, o que resulta na falta de robustez à dinâmica não modelada e distúrbios externos. Para aumentar a robustez no MRAC foi proposta uma lei de adaptação com modificação $\sigma$ (Ioannou e kokotovic (1984)), dada por

$$
\dot{\theta}=-\sigma \theta-\gamma e_{0} \omega, \quad \gamma>0 \text { e } \sigma>0
$$


onde o primeiro termo do lado direito da igualdade pode ser interpretado como um fator de esquecimento e o segundo termo, um fator de aprendizagem. Esta modificação garante no mínimo estabilidade local na presença de dinâmica não modelada e/ou distúrbios externos A introdução do fator $\sigma$, entretanto, pode levar ao aparecimento de "bursting" como verificado por Hsu e Costa (1987). Narendra e Anaswammi (1987) propuseram a utilização de um fator de esquecimento variável, substituindo o parâmetro sigma pelo módulo do erro de saída eliminando o efeito de "bursting.

A estabilidade global e a eliminação de "bursting" do algoritmo com modificação sigma, foi obtida depois por Ioannou e Tsakalis (1986), retendo a modificação sigma e introduzindo normalização no termo $e_{0} \omega$.

A introdução da normalização pode levar a transitórios de adaptação demasiadamente lentos e mesmo com a excitação rica em frequiência, a qualidade do transitório de adaptação (quando $\theta(t)$ está distante de $\theta^{*}$ ) não é uniforme e a convergência dos parâmetros adaptativos é muito lenta. Apesar do comportamento transitório não ser totalmente aceitável, em algumas situações, o sinal de controle é suave, tornando-o adequado para a condição de regime permanente.

\section{VS-MRAC}

O VS-MRAC foi desenvolvido por Hsu e Costa (1989), com o intuito de buscar um controlador robusto em relação às incertezas da planta e com um desempenho transitório significativamente melhor que os obtidos com os algoritmos baseados em identificação de parâmetros. Para o caso de $n^{*}=1$, a solução foi substituir as leis integrais de adaptação por leis de adaptação a estrutura variável, tornando o erro de saída $e_{0}(t) \equiv 0$ uma superfície deslizante no espaço de estado do erro do sistema e a condição de deslizamento é $\dot{e}_{0} e_{0}<0$. A lei de adaptação é dada por

$$
\theta_{i}=-\bar{\theta}_{i} \operatorname{sgn}\left(e_{0} \omega_{i}\right) \operatorname{com} \bar{\theta}_{i}>\left|\theta_{i}^{*}\right|, i=1,2 n
$$

$\bar{\theta}_{i}$ deve ser dimensionado de forma a levar em consideração as incertezas nos parâmetros da planta. No caso em que os parâmetros da planta sãoinvariantes no tempo pode-se demonstrar a estabilidade exponencial global para o VS-MRAC. Ainda, o algoritmo é estável para perturbações desconhecidas e uniformemente limitadas atuando na entrada da planta, desde que $\|\omega(t)\|$ seja maior que uma determinada constante positiva (Hsu e Costa (1989)) ( $\|\omega(t)\|$ é uma norma do vetor regressor $\omega(t)$ ). Simulações têm levado às mesmas conclusões para o caso variante no tempo. A grande desvantagem do VS-MRAC é que ele requer excessiva ação de controle e sua implementação prática pode apresentar algumas dificuldades.

\section{CONTROLADOR B-MRAC PARA N ${ }^{*}=1$}

A partir da teoria de controle binário desenvolvida por Emelyanov (1987), Hsu e Costa (1990) desenvolveram um esquema de controle denominado de Controle Binário Adaptativo por Modelo de Referência (B-MRAC). O B-MRAC consiste de uma lei gradiente de adaptação com projeção. A lei de adaptação é a mesma do MRAC com fator $\sigma$ (equação (13)) com a exceção de que $\sigma$ representa o fator de projeção especificado por

$$
\sigma=\left\{\begin{array}{l}
0, \text { se }\|\theta\|<M_{\theta} \text { ou } \sigma_{e q}<0, \\
\sigma_{e q}, \text { se }\|\theta\| \geq M_{\theta} \text { e } \sigma_{e q} \geq 0,
\end{array} \quad \sigma_{e q}=\frac{-\gamma e_{0} \theta^{T} \omega}{\|\theta\|^{2}}\right.
$$

$\operatorname{com} \gamma>0$ e $M_{\theta}>\left\|\theta^{*}\right\|$ é uma constante.

Com essa estrutura de controle mostra-se que se $\gamma \rightarrow \infty$, a lei de controle se torna

$$
\theta=-M_{\theta} \operatorname{sgn}\left(e_{0} \omega\right) \quad, \quad M_{\theta}>\left\|\theta^{*}\right\|
$$

que é a lei do VS-MRAC dada na equação (14). Para $\gamma$ suficientemente alto e sobre a condição de riqueza do sinal de referência, prova-se a estabilidade global exponencial uniforme do B-MRAC ( Hsu e Costa (1990) e (1994)). A exigência de alto ganho de adaptação para uma boa resposta transitória, pode, por sua vez, acarretar em amplificações nas perturbações e dinâmicas não modeladas, além de apresentar picos transitórios iniciais no sinal de controle em condições de elevado erro inicial de saída .

\section{CONTROLADOR EM MODO DUAL ADAPTATIVO ROBUSTO PARA $N^{*}=1$}

No controlador em Modo Dual Adaptativo Robusto é proposta uma ligação entre o MRAC e o VS-MRAC através de uma única lei de adaptação. Para a prova de estabilidade a lei de adaptação inicial (Cunha e outros, 2005) foi modificada. Basicamente, foi adicionado um termo na lei de controle de forma a se poder eliminar os termos de sinais indefinidos, que aparecem na derivada da função de Lyapunov. A idéia das ponderações da lógica nebulosa do tipo Takagi-Sugeno (Mota e Araújo (2002), Takagi e Sugeno (1985)) é incorporada em uma única expressão.

Considere a seguinte lei de adaptação

$$
\mu \dot{\theta}=-\sigma \theta-(1-\mu) \sigma \Gamma e_{0} \omega-\mu \gamma e_{0} \omega
$$

onde $\gamma>0, \sigma>0$ e $\Gamma=\operatorname{diag}\left[\frac{\bar{\theta}_{i}}{\left|e_{0} \omega_{i}\right|}\right], \bar{\theta}_{i}>\left|\theta_{i}^{*}\right|$, 
$i=1,2 n$.

Quando $\mu \rightarrow 0$, nota-se que a equação (17) se resume à equação (14), ou seja, ao algoritmo VS-MRAC. A equação (17) pode ser reescrita como

$\dot{\theta}=-\frac{\sigma}{\mu} \theta-\frac{1}{\mu}[(1-\mu) \sigma \Gamma+\mu \gamma I] e_{0} \omega, \quad \gamma>0$ e $\sigma>0$

Ainda, quando $\mu \rightarrow 0$, observa-se que o fator de esquecimento tende a infinito, implicando que o VS-MRAC não tem memória. O termo de aprendizagem também cresce ilimitadamente, de onde conclui-se que no VS-MRAC a adaptação é instantânea.

Quando $\mu=1$, a equação (17) se resume à (13) que é a lei de adaptação do MRAC com fator $\sigma$.

No DMARC (equação (18)) o fator de esquecimento é variável como em Narendra e Annaswamy (1987), e dependendo da forma como se ajusta o parâmetro $\mu$, o algoritmo DMARC pode trabalhar próximo do VS-MRAC. Esses fatores contribuem para o não surgimento do fenômeno de "bursting".

No algoritmo DMARC, o MRAC só começa a ter sua maior ponderação quando o erro de saída é suficientemente pequeno. Dessa forma e pelos fatores mencionados no final da seção 3, foi introduzido o termo $(1-\mu)$ na equação (17) de forma que a normalização (representada pelo termo $\Gamma$ ) é gradativamente reduzida, até se obter a lei com fator $\sigma$ que garante estabilidade local.

\section{PROVA DE ESTABILIDADE}

Considere o sistema e as hipóteses descritas na seção 2. Define-se o fator $\sigma$ como:

$$
\sigma= \begin{cases}0 & \text { se }\|\theta\|<M_{\theta} \text { e } \mu \neq 0 \\ \sigma_{c} & \text { em caso contrário }\end{cases}
$$

onde $M_{\theta}$ é dado por

$$
M_{\theta}=\|\bar{\theta}\|
$$

Teorema 7.1. Seja o sistema (5), (9), (17), (19) e uma bola $\|\theta\| \leq M_{\theta}$, definida como em (20). Então,

i) Todos os sinais em malha fechada são uniformemente limitados. ii) $\|e\| \rightarrow 0$ assintoticamente, se $\mu \rightarrow 0$

Prova: A prova de estabilidade é semelhante a apresentada em Hsu e Costa (1990). Escolhendo-se a função definida positiva como candidata a função de Lyapunov

$$
V(e, \tilde{\theta})=\frac{1}{2}\left(e^{T} P e+\frac{\tilde{\theta}^{T} \tilde{\theta}}{\theta_{2 n}^{*} \gamma}\right)
$$

Segundo o lema de Kalman-Yakubovitch $\left(A_{c}^{T} P+P A_{c}=\right.$ $-2 Q, P b_{c}=h_{c}, P=P^{T}>0$ e $\left.Q=Q^{T}>0\right)$ e, considerando que $e^{T} h_{c}=h_{c}^{T} e=e_{0}$, encontra-se para a derivada de (21)

$$
\dot{V}(e, \tilde{\theta})=-e^{T} Q e-\frac{\sigma \tilde{\theta}^{T}}{\theta_{2 n}^{*} \gamma \mu}\left[\theta+(1-\mu) \Gamma e_{0} \omega\right]
$$

que, juntamente com (17) resulta

$$
\begin{aligned}
\dot{V}(e, \tilde{\theta})=-e^{T} Q e- & \frac{\sigma}{\theta_{2 n}^{*} \gamma \mu} \sum_{i=1}^{2 n}\left[\theta_{i}-\theta_{i}^{*}\right] \\
& {\left[\theta_{i}+(1-\mu) \bar{\theta}_{i} \operatorname{sgn}\left(e_{0} \omega_{i}\right)\right] }
\end{aligned}
$$

Definindo os termos dentro do somatório como a função

$$
F_{i}\left(\theta_{i}\right)=\left[\theta_{i}-\theta_{i}^{*}\right]\left[\theta_{i}+(1-\mu) \bar{\theta}_{i} \operatorname{sgn}\left(e_{0} \omega_{i}\right)\right]
$$

vê-se que $\bar{\theta}_{i}$ e $-\bar{\theta}_{i}$ são as maiores raízes possíveis (em valores absolutos) de $F_{i}\left(\theta_{i}\right)$, pois $0<\mu \leq 1$ e $\bar{\theta}_{i}>\left|\theta_{i}^{*}\right|$.

Como o coeficiente de $\theta_{i}^{2}$ é positivo, então, $F\left(\theta_{i}\right)>0, \forall \theta_{i}$ tal que $\left|\theta_{i}\right|>\bar{\theta}_{i}$. Conseqüientemente, com (19) e (20) tem-se que

$$
\dot{V}(e, \tilde{\theta}) \leq-e^{T} Q e \leq 0
$$

De (21) e (24) conclui-se que $e, \tilde{\theta}$ e, conseqüentemente, $e_{0}$ e $\theta$ pertencem ao $L_{\infty}$. De $e=x_{c}-x_{c m}$, considerando que $r \in L_{\infty}$ e $M(s)$ é estável, tem-se que $x_{c m}$ e $y_{m} \in L_{\infty}$, consequentemente, $x_{c}$ e $y \in L_{\infty}$. Como $x_{c}^{T}=\left[x^{T}, v_{1}^{T}, v_{2}^{T}\right]$, tem-se que $x, v_{1}$ e $v_{2} \in L_{\infty}$. Desde

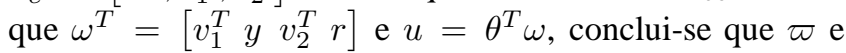
$u \in L_{\infty}$, resultando em i). 
Agora, se $\mu \rightarrow 0$, a equação (17) se resume à (14) e, no limite tem-se para (22) e (23)

$$
\dot{V}(e, \tilde{\theta})=-e^{T} Q e-\frac{\sigma}{\theta_{2 n}^{*} \gamma} \lim _{\mu \rightarrow 0} \sum_{i=1}^{2 n} \frac{1}{\mu} F_{i}\left(\theta_{i}\right)
$$

Fazendo o limite do termo dentro do somatório tem-se

$$
\begin{gathered}
\lim _{\mu \rightarrow 0} \frac{1}{\mu} F_{i}\left(\theta_{i}\right)=\lim _{\mu \rightarrow 0} \frac{1}{\mu}\left[\theta_{i}-\theta_{i}^{*}\right] \\
{\left[\theta_{i}+(1-\mu) \bar{\theta}_{i} \operatorname{sgn}\left(e_{0} \omega_{i}\right)\right]} \\
\lim _{\mu \rightarrow 0} \frac{1}{\mu} F_{i}\left(\theta_{i}\right)=\lim _{\mu \rightarrow 0} \frac{1}{\mu}\left[-\bar{\theta}_{i} \operatorname{sgn}\left(e_{0} \omega_{i}\right)-\theta_{i}^{*}\right] \\
{\left[(-\mu) \bar{\theta}_{i} \operatorname{sgn}\left(e_{0} \omega_{i}\right)\right]} \\
\lim _{\mu \rightarrow 0} \frac{1}{\mu} F_{i}\left(\theta_{i}\right)=\left[\bar{\theta}_{i}+\theta_{i}^{*} \operatorname{sgn}\left(e_{0} \omega_{i}\right)\right] \bar{\theta}_{i}>0
\end{gathered}
$$

pois $\bar{\theta}_{i}>\left|\theta_{i}^{*}\right|$. Dessa forma tem-se para (25)

$$
\dot{V}(e, \tilde{\theta})<-e^{T} Q e<0
$$

o que resulta em (ii).

\section{AJUSTE DO DMARC}

Para sintonizar o Controlador em Modo Dual Adaptativo Robusto utiliza-se uma expressão para o parâmetro $\mu$, a partir da idéia do modelo de Takagi-Sugeno (Mota e Araújo (2002)), dada pela equação (27) e ilustrada na Figura 1.

$$
\mu=e^{-e_{0}^{2} / L}
$$

onde $L$ é um parâmetro a ser ajustado.

Observa-se que quando $\mathrm{e}_{0} \rightarrow 0, \mu \rightarrow 1$ aproximando-se do MRAC. Quando $e_{0}$ se torna razoavelmente grande $\mu$ assume um valor suficientemente pequeno tendendo ao VS-MRAC. O parâmetro $L$ tem a importante função de determinar a forma como se dá a transição entre o MRAC e o VS-MRAC. Quanto menor o valor de $L$ maior a ação do VS-MRAC no espaço de $\mathrm{e}_{0}$.

A expressão (27) determina como o parâmetro $\mu$ deve ser variado, para ajustar o controlador DMARC, à medida que o erro e $e_{0}$ evolui. A derivada de $\mu$ é dada por

\begin{tabular}{|c|c|}
\hline $\begin{array}{c}e_{0} \dot{e}_{0}<0 \\
\text { condição de } \\
\text { escorregamento satisfeita }\end{array}$ & $\begin{array}{c}\dot{\mu}>0 \\
\mu \text { aumenta (aproxima-se } \\
\text { do MRAC) }\end{array}$ \\
\hline $\begin{array}{c}e_{0} \dot{e}_{0}>0 \\
\text { condição de } \\
\text { escorregamento não } \\
\text { satisfeita }\end{array}$ & $\begin{array}{c}\dot{\mu}<0 \\
\mu \text { diminui (aproxima-se } \\
\text { do VS-MRAC) }\end{array}$ \\
\hline $\begin{array}{c}e_{0} \dot{e}_{0}=0 \\
\text { superfície de } \\
\text { escorregamento alcançada }\end{array}$ & $\begin{array}{c}\dot{\mu}=0 \\
\mu \text { constante, controlador } \\
\text { fixo }\end{array}$ \\
\hline
\end{tabular}

$$
\dot{\mu}=-2 \frac{\mu}{L} e_{0} \dot{e}_{0}
$$

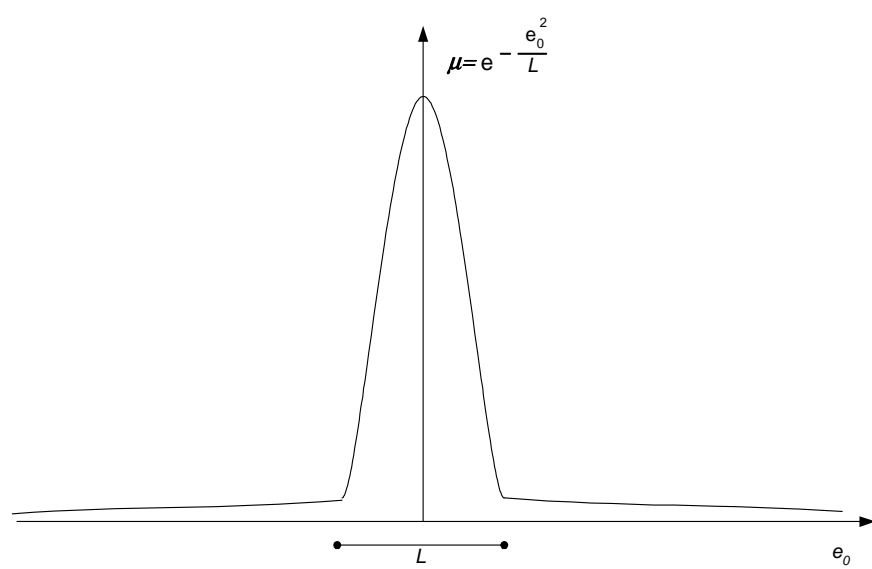

Figura 1: Evolução de $\mu$ função do erro de saída

Analisando essa derivada, considerando que $\mu$ é positivo, chega-se ao resultado apresentado na Tabela 1

Tabela 1: Variação de $\mu$ com a condição de escorregamento

Um aspecto prático da implementação do DMARC deve ser considerado a partir da observação da equação (17). Para evitar erro numérico, o valor de $\mu$, que se encontra no denominador de (18), é limitado inferiormente por um valor $\mu_{p}$, suficientemente pequeno.

Analizando-se ainda, a sintonia do DMARC, considere que o sistema está inicialmente operando como VS-MRAC, com $L$ e $\mu_{p}$ pequenos. Se após um determinado tempo a condição de deslizamento é satisfeita $\left(e_{0} \dot{e}_{0}<0\right)$ e o erro atinge um valor suficientemente pequeno (ver figura 1 ), a variação de $\mu$, expressa pela equação (28), será dada por

$$
\dot{\mu}=2 \frac{\mu_{p}}{L}\left|e_{0} \dot{e}_{0}\right|
$$

Considere agora, que o sistema está operando como MRAC com $L$ pequeno, $e_{0} \cong 0$ e $\mu \cong 1$. Se $e_{0}$ se afasta da origem tem-se $e_{0} \dot{e}_{0}>0$ (ver Figura 1) e desta forma a variação de $\mu$ será expressa por 


$$
\dot{\mu}=-2 \frac{1}{L}\left|e_{0} \dot{e}_{0}\right|
$$

Vê-se que a transição do VS-MRAC para o MRAC se dá de uma forma mais lenta (mais cautelosa) com uma taxa de variação $\mu_{p} / \mathrm{L}$ ( $\mu_{p}$ e $L$ pequenos), enquanto a transição do MRAC para o VS-MRAC, na mesma condição (mesmo valor absoluto de $e_{0} \dot{e}_{0}$ ), se dá com uma taxa de variação de aproximadamente $1 / \mathrm{L}$ ( $L$ pequeno).

Sendo $\mu_{p}$ suficientemente pequeno, o algoritmo de controle do DMARC é implementado com $\sigma$ dado por

$$
\sigma= \begin{cases}0 & \text { se }\|\theta\|<M_{\theta} \text { e } \mu>\mu_{p} \\ \sigma_{c} & \text { em caso contrário }\end{cases}
$$

onde $\frac{\sigma_{c}}{\mu_{p}}$ é suficientemente grande, tal que (17) seja o mais próximo possível de (15). Assim, se o erro de saída é tal que $\mu=\mu_{p} \mathrm{e}$, nesse caso, o DMARC atua como o VS-MRAC, espera-se, intuitivamente, que o erro de saída seja forçado a decrescer (note que no VS-MRAC $\|\theta\|=\|\bar{\theta}\|$ é constante). Dessa forma, teria-se $\mu_{p} \geq e^{-e_{0}^{2} / L}$. De uma forma mais relaxada, e considerando que esse é um resultado intuitivo tem-se

$$
\left|e_{0}\right|=O\left(\sqrt{-L \ln \left(\mu_{p}\right)}\right)
$$

A rigor, a exata convergência de (17) para (15) necessita que $\mu=0$. Porém, analisando (22), a condição necessária para que (26) seja satisfeita, é que $\frac{\sigma_{c}}{\mu_{p}}$ seja tal que $\left\|\theta^{*}\right\|<\left(1-\mu_{p}\right)\|\bar{\theta}\| \leq\|\theta\| \leq\|\bar{\theta}\|$.

\section{RESULTADOS DE SIMULAÇÕES}

Para ilustrar o funcionamento do DMARC, considere a planta de segunda ordem e grau relativo unitário expressa pela função de transferência

$$
W(s)=\frac{s+1}{(s-1)^{2}}
$$

O modelo de referência é especificado por

$$
M(s)=\frac{s+2}{(s+1)(s+3)}
$$

Foram escolhidos os filtros da entrada e saída como

$$
\Lambda=-2 \text { e } g=2
$$

Os parâmetros $\theta^{*}$ e $\bar{\theta}$ são respectivamente

$$
\theta^{*}=(0,5 ;-6 ; 5 ; 1)^{T} \text { e } \bar{\theta}=(0,6 ; 7 ; 6 ; 2)^{T}
$$

Nas simulações é utilizado o método de integração numérica de Euler. Inicialmente são feitas simulações com o intuito de observar as semelhanças existentes entre o controlador BMRAC e o controlador DMARC. Em ambos, o parâmetro de adaptação $\theta$ é limitado ao interior de uma bola de mesma dimensão $\left(M_{\theta}>\left\|\theta^{*}\right\|\right)$. No B-MRAC quando o vetor adaptado $\theta$, atinge a superfíce da bola ele é projetado sobre a mesma, permanecendo nela até que a lei de adaptação indique o decréscimo de $\|\theta\|$. No DMARC, quando o erro de saída é alto, a superfície da bola é atingida de forma chaveada, sendo essa, provavelmente, a principal diferença entre o DMARC e o B-MRAC.

Se o vetor de parâmetros de adaptaçao está no interior da bola, ou seja, $\|\theta\| \leq M_{\theta}$, tem-se nos dois controladores $\sigma=$ 0 e a lei de controle, tanto do DMARC (equações (17), (19) e (20)), como do B-MRAC (equações (13) e (15)) se resume a (12).

Nas primeiras simulações foram feitas as modificações na referência $r(t)$ e na perturbação $d(t)$ na entrada da planta, de acordo com a Tabela 2.

Tabela 2: Variações em $r$ e $d$ durante as simulações

\begin{tabular}{|c|c|c|}
\hline INTERVALO & REFERÊNCIA & PERTURBAÇÃO \\
\hline $0 \leq t<0,8$ & $r(t)=1$ & $d(t)=0,8$ \\
\hline $0,8 \leq t<1,6$ & $r(t)=-1$ & $d(t)=0,0$ \\
\hline $1,6 \leq t<2,4$ & $r(t)=1$ & $d(t)=0,8$ \\
\hline
\end{tabular}
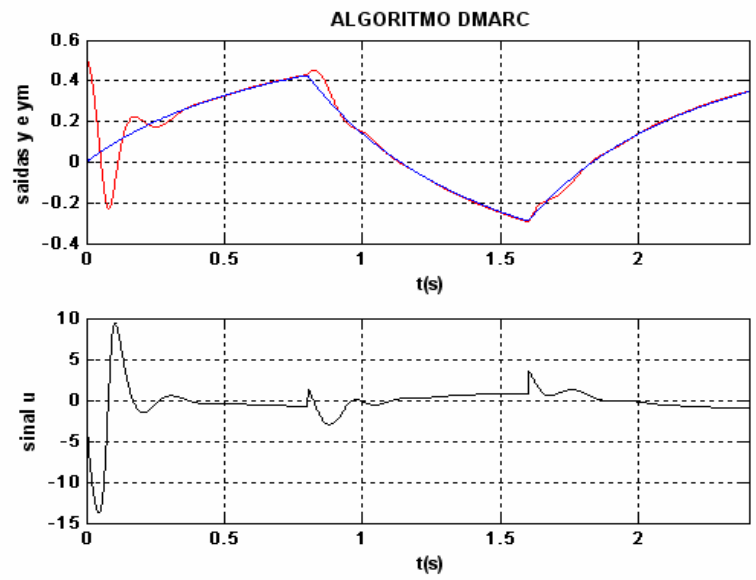

Figura 2a: Resultado do controlador DMARC

Com o mesmo valor de $\gamma=750$ e sob as mesmas condições foi feita a simulação com o B-MRAC e o resultado encontra- 


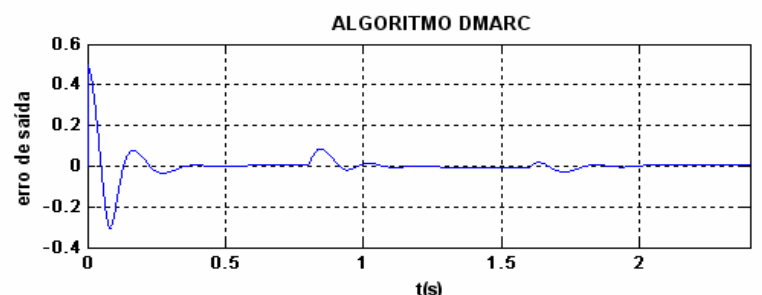

t(s)

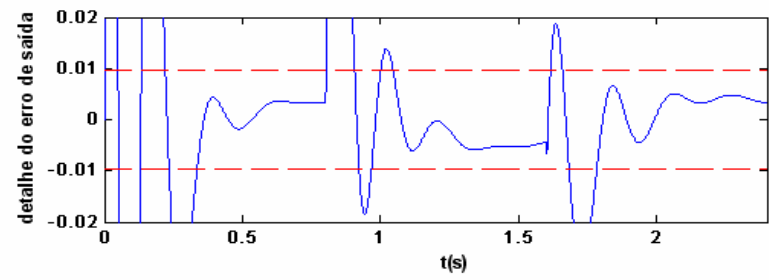

Figura 2b: Resultado do controlador DMARC

se na Figura 3. A semelhança entre os resultados obtidos nas figuras 2a e 3, se deve ao fato de que, com gama grande e com uma relação $\frac{\sigma_{c}}{\mu_{p}}$ de valor não suficientemente alto, o DMARC, semelhantemente ao B-MRAC, trabalha como um MRAC com uma lei gradiente de adaptação de alto ganho. $\mathrm{Na}$ Figura $2 \mathrm{~b}$ a linha pontilhada no detalhe do erro de saída, representa o limite do erro dado pela equação (32).
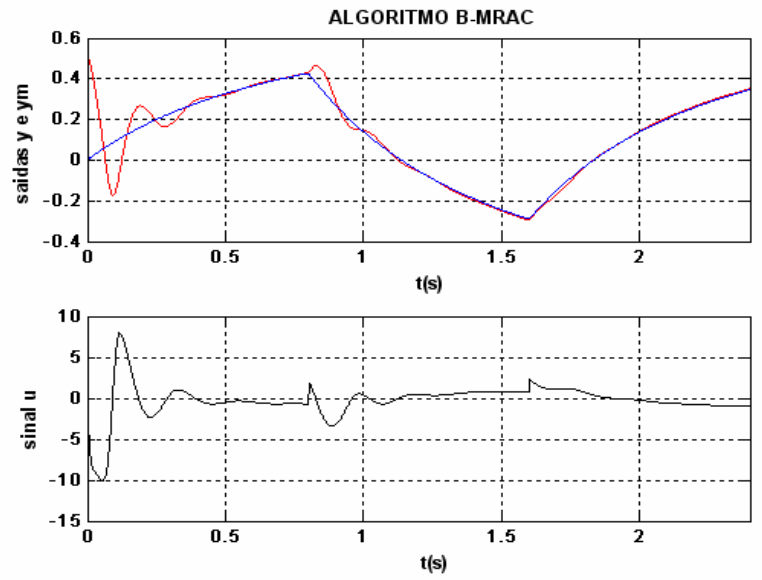

Figura 3 : Resultado do controlador B-MRAC

Para comparar os resultados do B-MRAC, VS-MRAC e o DMARC, novas simulações foram feitas, sob as condições apresentadas na Tabela 3 .

Tabela 3: Variações em $r$ e $d$ durante as simulações

\begin{tabular}{|c|c|c|}
\hline INTERVALO & REFERÊNCIA & PERTURBAÇ̃̃̃ \\
\hline $0 \leq t<0,2$ & $r(t)=1$ & $d(t)=0,8$ \\
\hline $0,2 \leq t<0,4$ & $r(t)=-1$ & $d(t)=0,0$ \\
\hline $0,4 \leq t<0,6$ & $r(t)=1$ & $d(t)=0,8$ \\
\hline
\end{tabular}

Para obter uma resposta rápida no B-MRAC foram utilizados $h=10^{-5}$ s e $\gamma=10^{6}$ e o resultado é apresentado nas figuras $4 \mathrm{a}$ e $4 \mathrm{~b}$.
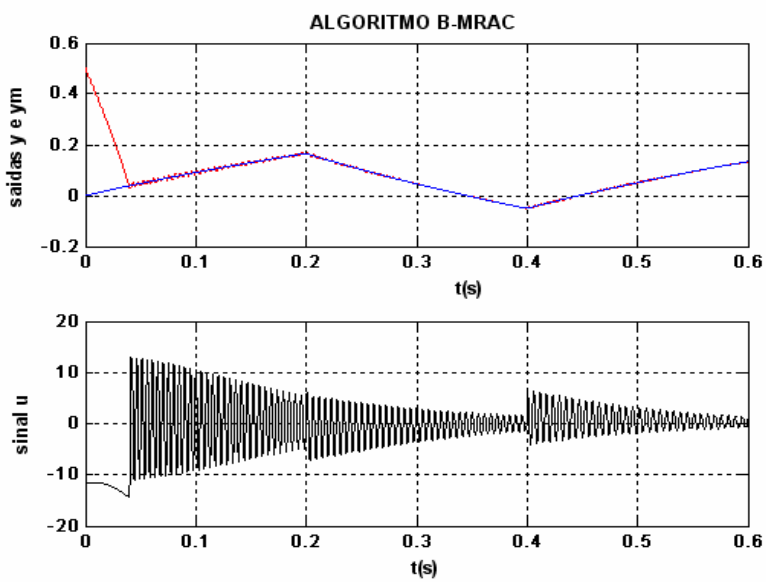

Figura 4a: Resultado do controlador B-MRAC

Na figura 4a, observa-se que no B-MRAC, com $\gamma$ grande, a saída da planta se aproxima da saída do modelo de referência mais rapidamente que nos casos do VS-MRAC e DMARC (Figuras 5a e 6a, respectivamente). No entanto, o B-MRAC tem um sinal de controle, inicial, de maior amplitude. Além disso, o BMRAC, apesar de $\gamma$ grande, apresenta oscilações de maior amplitude (Figura 4b) que o VS-MRAC (Figura 5b) e o DMARC (Figura 6b). Na Figura 4b, a linha pontilhada, no detalhe do erro de saída do B-MRAC, serve apenas para comparação com o DMARC das Figuras 7a e 7b.
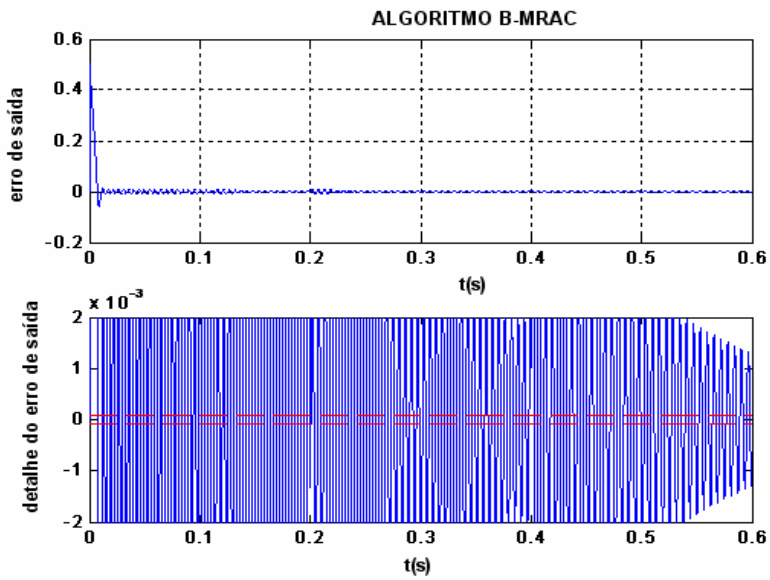

Figura 4b: Resultado do controlador B-MRAC

Para o VS-MRAC foi usado $h=10^{-5} s$ e os resultados são apresentados nas figuras $5 \mathrm{a}$ e $5 \mathrm{~b}$.

No VS-MRAC, para se obter um modo deslizante, é necessário que a frequência de chaveamento seja alta, o que leva ao uso de um passo de integração muito pequeno. Considerando 


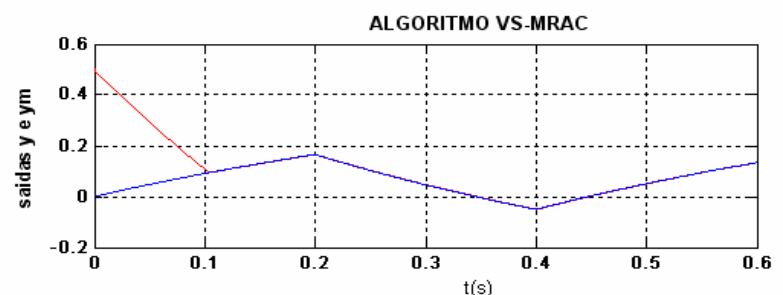

$t(s)$

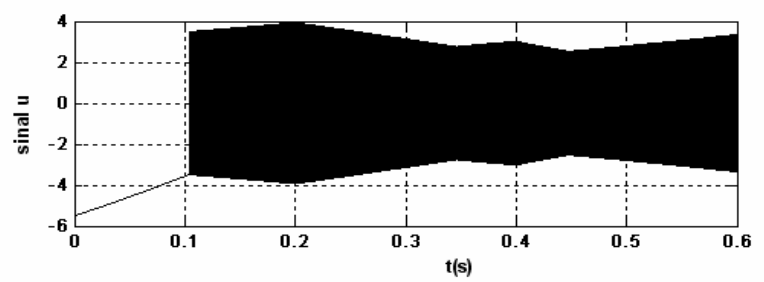

Figura 5a: Resultado do controlador VS-MRAC
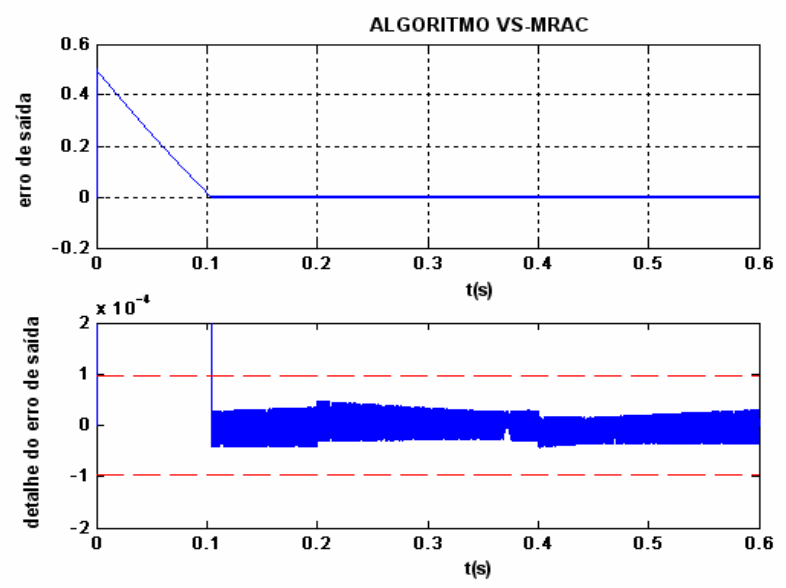

Figura 5b: Resultado do controlador VS-MRAC

que $h=10^{-5} s$ é uma boa escolha para o bom funcionamento do VS-MRAC, são feitas simulações com o DMARC usando $h=10^{-5} s, L=10^{-9}, \gamma=10, \sigma_{c}=10^{-1}$, $\mu_{p}=10^{-4}$ e são obtidos os resultados apresentados nas Figuras $6 \mathrm{a}$ e $6 \mathrm{~b}$. O valor de $\gamma$ foi reduzido, em relação à primeira simulação (Figura 2a e 2b), pois no DMARC o funcionamento como o VS-MRAC pode ser especificado pelos parâmetros $L, \sigma_{c}$ e $\mu_{p}$, ao passo que no B-MRAC o funcionamento como VS-MRAC só é possível com $\gamma$ tendendo para infinito (ver Hsu e Costa (1990) e (1994)). Ainda, $\gamma$ muito alto pode amplificar os efeitos de perturbações externas e dinâmicas não modeladas.

Da Figura 6a, observa-se que a planta segue o modelo praticamente sem oscilações, uma característica do VS-MRAC, e o sinal de controle apresenta uma certa suavidade em relação ao chaveamento do VS-MRAC. Na Figura 6b é apresentado o erro de saída da simulação referente à Figura 6a, a linha pontilhada, no detalhe do erro de saída representa o limite dado pela equação (32). O controle apresenta robustez com
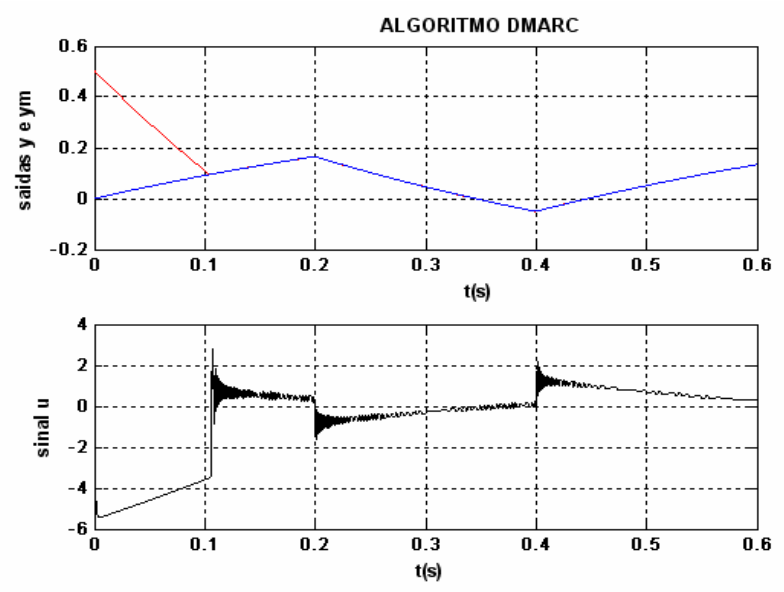

Figura 6a: Resultado do controlador DMARC
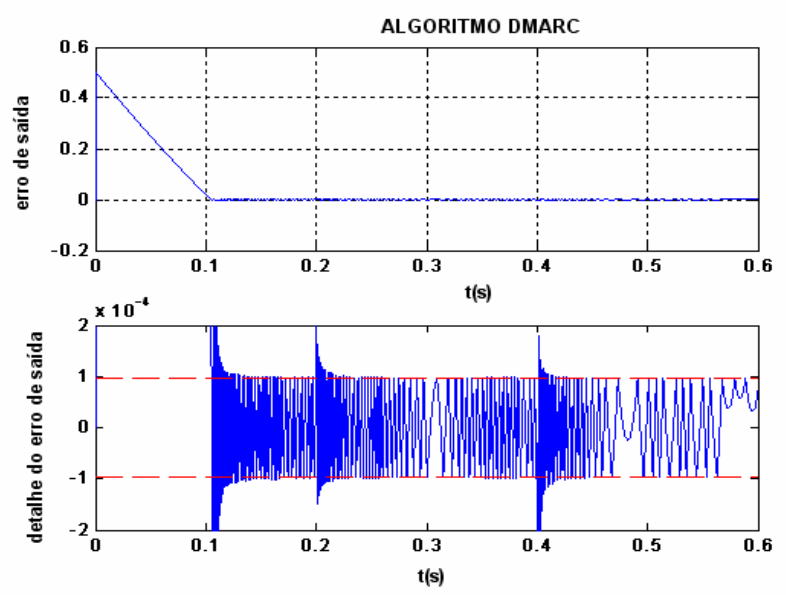

Figura 6b: Resultado do controlador DMARC

relação a perturbações externas na entrada da planta.

Para atenuar mais o sinal de controle, pode-se diminuir a região de ação do VS-MRAC, em relação ao erro de saída, aumentando-se o valor de $L$. O valor de $h$, nesse caso, pode ser aumentado. Então com $h=10^{-4} s, L=10^{-7}, \gamma=10$, $\sigma_{c}=10^{-1}, \mu_{p}=10^{-4}$, nova simulaçao é feita com o DMARC e o resultado é apresentado nas Figuras $7 \mathrm{a}$ e $7 \mathrm{~b}$.

Pode-se observar na Figura 7a uma boa suavização do sinal de controle, em relação ao sinal apresentado na Figura 6a. Porém, na Figura $7 b$, vê-se que o erro de saída agora é da ordem de $10^{-3}$, enquanto que o da Figura $6 \mathrm{~b}$ é da ordem de $10^{-4}$.

Com uma relação $\frac{\sigma_{c}}{\mu_{p}}$ suficientemente grande e com $L$ bem pequeno, o DMARC trabalha como VS-MRAC até que o erro de saída seja bem próximo de zero (ver Figura 1), levando o sistema a uma resposta transitória consideravelmente rápida. Obviamente, se trabalharmos com $L \cong 0$, ape- 


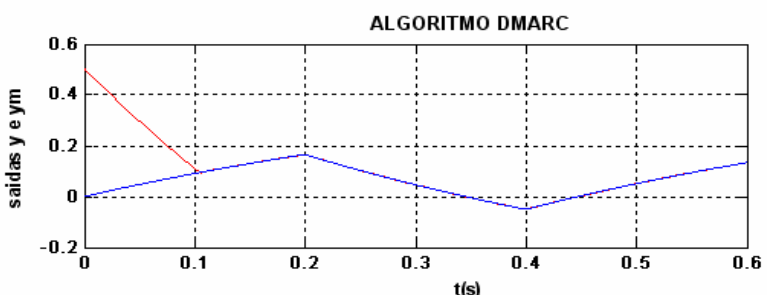

$t(s)$

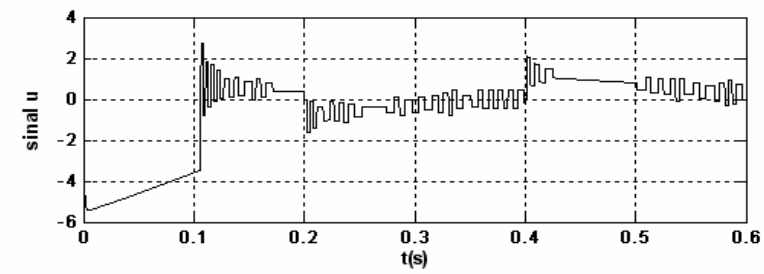

Figura 7a: Resultado do controlador DMARC
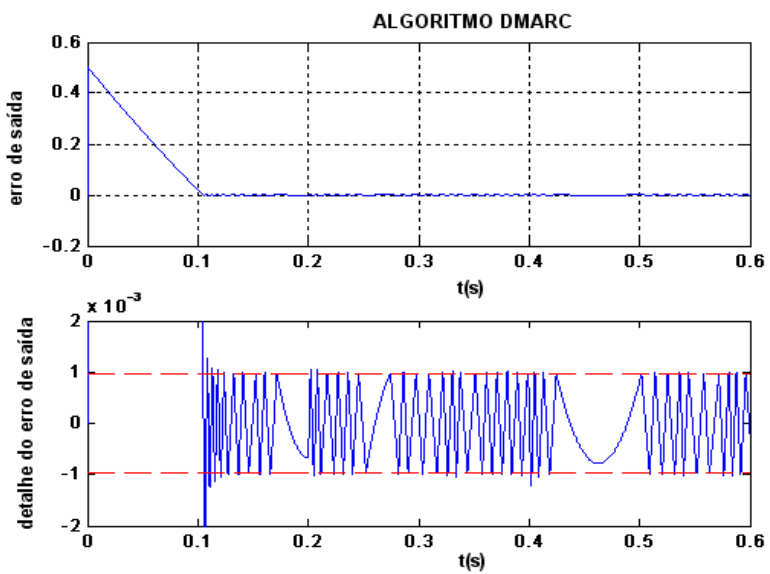

Figura 7b: Resultado do controlador DMARC

sar de se ter uma boa resposta transitória, pode-se ter um sinal de controle com uma frequiência elevada. Assim, a escolha dos parâmetros $L, \sigma_{c}$ e $\mu_{p}$ irá ditar o compromisso entre resposta transitória rápida e suavidade no sinal de controle.

\section{CONCLUSÕES}

Neste artigo é apresentada uma demonstração de estabilidade para o controlador em Modo Dual Adaptativo Robusto para plantas com grau relativo unitário. Conforme pode ser verificado pelas simulações, o algoritmo em Modo Dual Adaptativo Robusto proporcionou um transitório rápido e praticamente sem oscilações e um desempenho em regime permanente com sinal de controle com boa suavização. Adicionalmente apresentou robustez a incertezas paramétricas e distúrbios.

Para plantas com grau relativo arbitrário, o DMARC utiliza a mesma estrutura do VS-MRAC, usando a lei de adaptação do DMARC, para $n^{*}=1$, no bloco onde é gerado o sinal de controle que será aplicado à planta (Cunha e Araújo (2004)). A análise de estabilidade, para plantas $\operatorname{com} n^{*}>1$, pode ser feita a partir da análise de plantas com $n^{*}=1$, seguindo Hsu (1990) e Hsu e Costa (1994).

\section{REFERÊNCIAS}

Åström, K. J. e Wittenmark, B. (1995). Adaptive Control, Addison-Wesley Pub. Co.

Cunha, C. D., Araújo, A. D., Barbalho, D. S. e Mota, F. C. (2005). A Dual-Mode Adaptive Robust Controller Applied to the Speed Control of a Three-Phase Induction Motor, Asian Journal of Control, 7(2), 197-201.

Cunha, C. D. e Araújo, A. D. (2004). A Dual-Mode Adaptive Robust Controller for Plants with Arbitrary Relative Degree. Proceedings of 8th International Workshop on Variable Structure Systems - VSS'04. Vilanova i la Geltrú, Espanha.

Cunha, J.P.V.S., Hsu, L., Costa, R.R. e Lizarralde, F. (2003). Output-Feedback Model-Reference Sliding Mode Control of Uncertain Multivariable Systems, IEEE Transactions on Automatic Control, AC-48 (12), 2245-2250.

Emelyanov, S. V. (1987). Binary Automatic Control Systems. MIR Publlishers, Moscow (English Translation).

Hsu, L. and Costa, R. R. (1987), "Bursting Phenomena in Continuous-Time Adaptive Systems with a SigmaModification", IEEE Trans. Automatic Control, AC-32 (1), 84-86.

Hsu, L. e Costa, R. R. (1989). Variable Structure Model Reference Adaptive Control Using only Input and Output Measurements: Part I, Int. J. Control, 49(2), 399-416.

Hsu, L., Araújo, A. D. e Costa, R. R. (1994). On the Design of Variable Structure Adaptive Control Using only Input/ Output Data. IEEE Trans. Automatic Control, AC39 (1), 4-21.

Hsu, L. e Costa, R.R. (1990). A Binary Control Approach to Design Globally Exponentially Stable Systems. Proceedings of the $7^{\text {th }}$ International Conference on Systems Engineering, Las Vegas, July.

Hsu, L. e Costa, R. R. (1994). B-MRAC Global Exponential Stability with a New Model Reference Adaptive Controller Based on Binary Control Theory. ControlTheory and Advanced Technology, 10 (4), Part 1, 649668.

Hsu, L., Lizarralde, F. e Araujo, A. D. (1997). New Results on Output-Feedback Variable Structure ModelReference Adaptive Control: Design and Stability Analysis, IEEE Transactions AC-42 (3), 386-393 
Ioannou, P. A. e Sun, J. (1996). Robust Adaptive Control, Prentice-Hall, Inc., Englewood Cliffs.

Ioannou, P. A. e Kokotovic, P. V. (1984). Instability Analysis and Improvement of Robustness of Adaptive Control. Automatica, AC-20(5),583-594

Ioannou, P. A. e Tsakalis, K. S. (1986). A Robust Direct Adaptive Controller. IEEE Trans. Automatic Control, AC-31( 11), 1033-1043.

Mota, F. C. e Araújo, A. D. (2002). Proposta de um Controlador Dual Adaptativo Robusto Utilizando o Modelo de Takagi-Sugeno, Congresso Brasileiro de Automática Natal-RN, Brasil, 173-177.

Narendra, K. S. e Annaswamy, A. M. (1987). New Adaptive Law for Robust Adaptive Control Without Persistent Excitation, IEEE Trans Automatic Control, AC-32, $134-145$.

Narendra, K. S. e Annaswamy, A. M. (1988). Stable Adaptive Systems, Prentice Hall, Inc., New Jersey.

Sastry, S. S. (1984). Model - Reference Adaptive Control Stability, Parameter Convergence and Robustness, IMA J. Mathematical Control and Information, 1, 27-66.

Sastry, S. S. e Bodson, M. (1989). Adaptive Control: Stability Convergence and Robustness, Englewood Cliffs, NJ, Prentice-Hall.

Takagi, T e Sugeno, M. (1985). Fuzzy Identification of Systems and its Applications to Modeling and Control, IEEE Trans. Syst., Man, Cybern. SMC-15(1), 116-132. 